\title{
MicroRNA-195 acts as a tumor suppressor by directly targeting Wnt3a in HepG2 hepatocellular carcinoma cells
}

\author{
YANG YANG $^{1 *}$, MENGHE LI $^{1 *}$, SU'E CHANG $^{1}$, LUMIN WANG $^{1}$, TUSHENG SONG $^{1}$, LING GAO $^{1}$, \\ LILI HU $^{1}$, ZONGFANG LI ${ }^{2}$, LIYING LIU ${ }^{1}$, JIAYI YAO ${ }^{1}$ and CHEN HUANG ${ }^{1,3,4}$ \\ ${ }^{1}$ Department of Genetics and Molecular Biology, Xi'an Jiaotong University College of Medicine; \\ ${ }^{2}$ Engineering Research Center of Biotherapy and Translational Medicine of Shaanxi Province; \\ ${ }^{3}$ Key Laboratory of Environmentally and Genetically Associated Diseases at Xi'an Jiaotong University, \\ Ministry of Education; ${ }^{4}$ Cardiovascular Research Center, Xi'an Jiaotong University College of Medicine, \\ Xi'an, Shaanxi 710061, P.R. China
}

Received November 28, 2013; Accepted July 1, 2014

DOI: $10.3892 / \mathrm{mmr} .2014 .2526$

\begin{abstract}
MicroRNAs (miRNAs) are a class of small, non-coding, endogenous RNAs that are important in tumor cell biological processes as they regulate gene expression. miR-195 has been demonstrated to be a tumor repressor in numerous types of human cancer. However, the mechanism by which miR-195 suppresses tumor development remains to be elucidated. The aim of this study was to investigate the effect of miR-195 on the biological functions of HepG2 hepatocellular carcinoma (HCC) cells and identify the association between miR-195 and Wnt3a in HCC. miR-195 mRNA expression levels in HCC tissues and cell lines were measured by reverse transcription polymerase chain reaction analysis. miR-195 function was measured with cell proliferation, cell cycle and apoptosis assays following transfection with miR-195 and anti-miR-195 sequences, and the respective controls. Luciferase reporter assay was used to determine whether Wnt3a was a target of miR-195. In addition, Wnt3a expression levels were determined in HCC cells using western blot analysis. The miR-195 expression levels were found to be reduced in HCC tissues and cell lines. miR-195 overexpression resulted in a reduction in cell proliferation. In addition, the overexpression of miR-195 in HCC cells induced G1 phase
\end{abstract}

Correspondence to: Mr. Chen Huang, Key Laboratory of Environmentally and Genetically Associated Diseases at Xi'an Jiaotong University, Ministry of Education, 76 West Yanta Road, Xi'an, Shaanxi 710061, P.R. China

E-mail: hchen@mail.xjtu.edu.cn

Miss. Jiayi Yao, Department of Genetics and Molecular Biology, Xi'an Jiaotong University College of Medicine, 76 West Yanta Road, Xi'an, Shaanxi 710061, P.R. China

E-mail: yaojiayi@mail.xjtu.edu.cn

${ }^{*}$ Contributed equally

Key words: hepatocellular carcinoma, microRNA-195, proliferation, Wnt3a cell cycle arrest and promoted apoptosis. Furthermore, Wnt3a was demonstrated to be directly targeted by miR-195. These findings suggest that miR-195 is key in regulating cell proliferation, cell cycle and apoptosis through targeting Wnt3a. In addition, overexpression of miR-195 may be a potential therapeutic strategy in the treatment of $\mathrm{HCC}$.

\section{Introduction}

Liver cancer is the fifth most common type of malignancy worldwide and the third most common cause of cancer-related mortality. As an aggressive disease with a poor outcome, liver cancer results in $>600,000$ fatalities per year $(1,2)$. In total, $\sim 90 \%$ of all primary liver cancer diagnoses are classified as hepatocellular carcinoma (HCC). Therefore, for the majority of the time, the terms liver cancer and HCC may be used interchangeably (3). Studies have found that 70-90\% HCC cases are caused by liver cirrhosis; the predominant risk factor for $\mathrm{HCC}$ worldwide is chronic hepatitis B virus infection (4). HCC development occurs in multiple stages, which include genetic and epigenetic changes, the activation of oncogenes or the inactivation of tumor suppressor genes in cancer cells.

MicroRNAs (miRNAs) are a class of small, non-coding RNAs that regulate gene expression through targeted inhibition of transcription and translation. Recent studies have suggested that miRNAs are important in tumor cell biological processes, including cell proliferation, cell cycle, apoptosis, migration and invasion (5-7). Furthermore, miRNAs have emerged as potential therapeutic agents for tumorigenesis due to the ability to downregulate multiple targets, not only in tumor progression and metastasis, but also in therapeutic resistance and tumor recurrence (8). In HCC carcinogenesis and progression, certain miRNAs have been reported to be dysregulated, for example, miR-135, miR-21 and miR-17-92 are upregulated, and miR-122, miR-125, miR-375, miR-101 and miR-150 are downregulated (6,9-14). Recently, miR-195 has been reported to exert vital functions in a number of types of cancer, including bladder, adrenocortical, breast and gastric cancer (15-18); thus, miR-195 may be critical in cancer development. The aim of this study was to investigate the effect of miR-195 on the biological 
function of HepG2 cells and to identify the correlation between miR-195 and Wnt3a in HCC.

\section{Materials and methods}

Human tissue samples and cell lines. Surgically removed HCC tumor tissues and matched adjacent non-cancerous tissues used for reverse transcription polymerase chain reaction (RT-PCR) and western blotting were obtained from $28 \mathrm{HCC}$ patients at the First Affiliated Hospital of Xi'an Jiaotong University College of Medicine (Xi'an, China). No local or systemic treatment had been conducted prior to surgery. The SMMC-7721, HepG2, Hep3B and Bel-7402 liver cancer cell lines and the HL-7702 normal liver cell line were obtained from the Key Laboratory of Environmentally and Genetically Associated Diseases at Xi'an Jiaotong University, Ministry of Education (Xi'an, Shaanxi, China). The cells were maintained in Dulbecco's modified Eagle's medium (PAA Laboratories, Pasching, Austria) supplemented with 10\% fetal bovine serum. All cells were incubated at $37^{\circ} \mathrm{C}$ in $5 \% \mathrm{CO}_{2}$. The study was approved by the Medical Ethical Committee of the College of Medicine, Xi'an Jiaotong University, Xi'an, China and all patients provided written informed consent.

RNA extraction and RT-PCR. Total RNA, including miRNA from tissue samples and cells was isolated using TRIzol reagent (Invitrogen Life Technologies, Carlsbad, CA, USA). The concentration of RNA was measured using a NanoDrop ND-1000 spectrophotometer (NanoDrop Technologies, Wilmington, DE, USA). The mRNA was reverse transcribed using a reverse transcription kit (Takara Biotechnology Co., Ltd., Dalian, China), according to the manufacturer's instructions. The relative levels of miR-195 were examined using altered stem-loop RT-PCR with specific RT and PCR primers; U6 served as a control. The reverse transcription primers of miR-195 and U6 are listed in Table I. Relative quantification of mRNA expression levels was performed according to the manufacturer's instructions (Bio-Rad, Hercules, CA, USA).

Plasmid constructs. The precursors of the hsa-miR-195 sequence were synthesized with the following EcoRI and HindIII top oligo: 5'-AATTCAGCTTCCCTGGCTCTAGCAGCACAGAAATATTGGCACAGGGAAGCGAGTCTGCCAATATTGGCTGTGCTGCTCCAGGCAGGGTGGTGA-3'; and the following bottom oligo: 3'-GTCGAAGGGACCGAGATCGTCGTGTCTTTATAACCGTGTCCCTTCGCTCAGACG GTTATAACCGAACACGACGAGGTCCGTCCCACCACTT CGA-5'. Then the miR-15b was inserted into the EcoRI and HindIII sites of the pcDNA $^{\mathrm{TM}}$ 6.2-GW/EmGFP-miR vector (National Center for Biotechnology Information; http://www. ncbi.nlm.nih.gov/).

MTT cell proliferation and colony forming assays. A total of $5 \times 10^{3} \mathrm{HepG} 2$ cells were seeded in 96-well culture dishes, and incubated for 24,48 or $72 \mathrm{~h}$ at $37^{\circ} \mathrm{C}$ in a humidified incubator with $5 \% \mathrm{CO}_{2}$. Subsequently, $20 \mu 1 \mathrm{MTT}$ was added to the cells and the cells were incubated for $4 \mathrm{~h}$. The supernatant was removed and replaced with $150 \mu \mathrm{l}$ dimethyl sulfoxide and the cells were then incubated at $37^{\circ} \mathrm{C}$ in a humidified incubator with $5 \% \mathrm{CO}_{2}$. The absorbance was measured at $490 \mathrm{~nm}$ using the FLUOstar OPTIMA (BMG Labtech, Ortenberg, Germany). To
Table I. miR-195 and U6 forward and reverse primers for reverse transcription polymerase chain reaction.

miRNA Primer

miR-195 RT: GTCGTATCCAGTGCGTGTCGTGGAGTC GGCAATTGCACTGGATACGACGCCAATA Forward: ATCCAGTGCGTGTCGTG Reverse: TGCTTAGCAGCACAGAAA

U6 RT: CGCTTCACGAATTTGCGTGTCAT Forward: GCTTCGGCAGCACATATACTAAAAT Reverse: CGCTTCACGAATTTGCGTGTCAT

miRNA, microRNA; RT, reverse transcription.

assess cell colony formation ability, $5 \times 10^{2}$ HepG2 cells transfected with either miR-195, miR-ctrl, anti-miR-195 or anti-ctrl were seeded in 6-well culture dishes. After 14 days incubation at $37^{\circ} \mathrm{C}$ in a humidified incubator with $5 \% \mathrm{CO}_{2}$, the cells were washed twice with phosphate-buffered saline (PBS) and stained with crystal violet solution. Images were obtained using Quantity One computer software (Bio-Rad). All assays were repeated three times.

Luciferase activity assay. A fragment of the 3' untranslated region (UTR) of Wnt3a was synthesized with SacI and XhoI restriction enzymes using the following primers: Wnt3a, 5'-CGCGAGTCTGCCAATATTGGCTGTGCTGC TCCAGGCAGGGTGGTGC-3'; and Wnt3a mutation, 5'-CGCGAGTCTGCCAATATTGGCTGAGGTGATCCAGG CAGGGTGGTGC-3'. HEK293 cells were obtained from the Key Laboratory of Environmentally and Genetically Associated Diseases at Xi'an Jiaotong University, Ministry of Education (Xi'an, China). A total of $1 \times 10^{4}$ HEK293 cells were seeded into each well of a 96-well plate, subsequent to pmirGLO-Wnt3a-3'UTR-wt or pmirGLO-Wnt3a-3'UTR-mut vector co-transfection with miR-195 or miR-ctrl for $24 \mathrm{~h}$ using Lipofectamine 2000 (Invitrogen Life Technologies). Firefly luciferase activity was measured at $24 \mathrm{~h}$ post-transfection using a Dual-Luciferase Reporter Assay system (Promega Corporation, Madison, WI, USA). The results were normalized with Renilla luciferase. Each reporter plasmid was transfected at least three times and each sample was assayed in triplicate.

Cell cycle analysis. HepG2 cells were transfected with miR-195, miR-ctrl, anti-miR-195 or anti-ctrl when the cells had grown to $70-90 \%$ confluence. The cells were then incubated at $37^{\circ} \mathrm{C}$ for $48 \mathrm{~h}$. Subsequently, the treated cells were collected and fixed in $70 \%$ ethanol at $4{ }^{\circ} \mathrm{C}$ overnight. The cells were then washed in PBS and incubated with $1 \mathrm{ml}$ staining solution $(20 \mu \mathrm{g} / \mathrm{ml}$ propidium iodide and $10 \mathrm{U} / \mathrm{ml}$ RNase A) for $30 \mathrm{~min}$ at room temperature.

The cell-cycle distributions were assayed by fluorescence-activated cell sorting using a flow cytometer (FACSort; Becton-Dickinson, Franklin Lakes, NJ, USA).

Apoptosis analysis. HepG2 cells were transfected with miR-195, miR-ctrl, anti-miR-195 or anti-ctrl when the cells had 
A

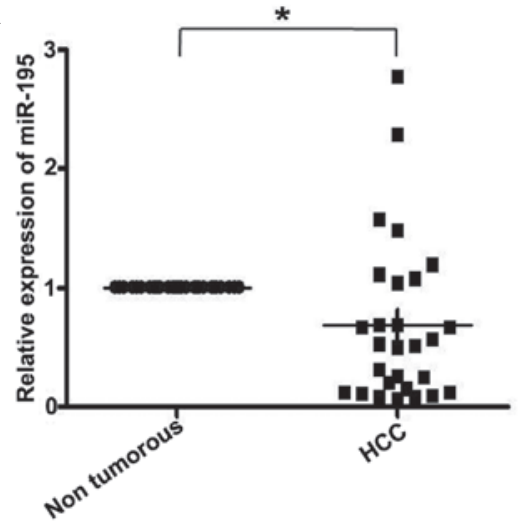

B

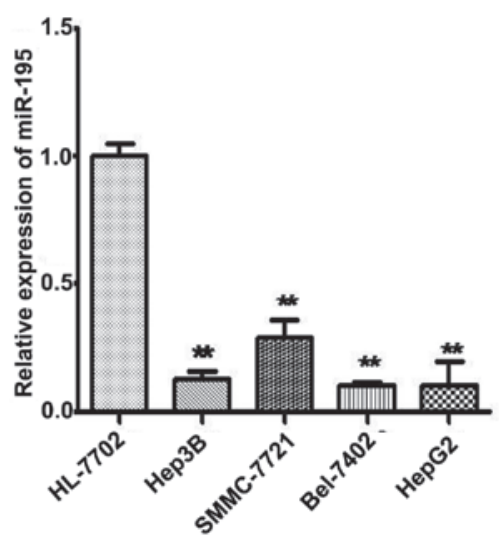

Figure 1. Expression levels of miR-195 in HCC tissues and cell lines. (A) miR-195 expression levels were detected in 28 paired HCC tissues using reverse transcription polymerase chain reaction; U6 served as an internal control. (B) Relative levels of miR-195 expression in the HCC cell lines. Results signify mean \pm standard deviation $(\mathrm{n}=3)$. ${ }^{*} \mathrm{P}<0.05,{ }^{* *} \mathrm{P}<0.01$. miR, microRNA; HCC, hepatocellular carcinoma.

grown to $70-90 \%$ confluence. The cells were cultured at $37^{\circ} \mathrm{C}$ for $48 \mathrm{~h}$ then harvested by trypsinization and centrifugation, and washed twice with PBS. The cells were then stained using an Annexin V/fluorescein isothiocyanate Apoptosis Detection kit (Invitrogen Life Technologies) according to the manufacturer's instructions. Cell apoptosis were examined using a flow cytometer (Becton-Dickinson).

Western blot analysis. Either miR-195 or anti-miR-195 was transfected into HepG2 cells using Lipofectamine 2000, with miR-ctrl and anti-ctrl serving as respective controls. Total proteins were isolated from the cells by radioimmunoprecipitation assay $48 \mathrm{~h}$ post-transfection. Protein concentrations were measured using a micro bicinchoninic acid protein assay kit (Pierce Biotechnology, Inc, Rockford, IL, USA). The proteins were resolved using a $10 \%$ SDS-PAGE gel, transferred onto the nitrocellulose membrane, blocked in 5\% non-fat dry milk in Tris-buffered saline ( $\mathrm{pH} 7.4$ ) containing $0.05 \%$ Tween 20 (TBST) and incubated with rabbit polyclonal antibody against Wnt3a overnight at $4^{\circ} \mathrm{C}$ (1:200; Beijing Biosynthesis Biotechnology Co., Ltd., Beijing, China). After washing three times with TBST, the membrane was incubated with a goat anti-rabbit IgG (1:5,000; Santa Cruz Biotechnology, Inc., Santa Cruz, CA, USA) antibody for $2 \mathrm{~h}$, then washed three times with TBST. GAPDH served as a control. Relative protein expression was measured on the Quantity One imaging software. All western blot analyses were performed three times.

Statistical analysis. All data are expressed as the mean \pm standard deviation. Student's t-test was used for statistical analysis. $\mathrm{P}<0.05$ and $\mathrm{P}<0.01$ were considered to indicate statistically significant differences. All analyses were performed using SPSS 13.0 software (SPSS, Inc., Chicago, IL, USA).

\section{Results}

miR-195 exhibits low-level expression in HCC tissues and cell lines. The RT-PCR detection results revealed that the expression levels of miR-195 were significantly downregulated in the 28 pairs of HCC tissues in comparison with matched non-tumor tissues from patients $(\mathrm{P}<0.05$; Fig. 1A). Furthermore, miR-195 was found to be significantly downregulated in the Bel-7402, SMMC-7721, HepG2 and Hep3B HCC cells compared with the HL-7702 normal hepatocyte cells $(\mathrm{P}<0.01$; Fig. 1B). These results reveal that miR-195 is downregulated in HCC tissues and cell lines. The reduced expression levels of miR-195 in HCC suggest that miR-195 is a potential antioncogenic miRNA in HCC and miR-195 downregulation may be involved in human HCC development.

miRNA-195 regulates the proliferation and colony formation of liver cancer cells. To analyse the function of miR-195 in cell growth, miR-195-transfected HepG2 cells were compared with cells transfected with miR-ctrl, anti-miR-195 and anti-ctrl. As demonstrated by MTT growth assays, overexpression of miR-195 significantly reduced cell proliferation compared with the miR-ctrl group. Conversely, anti-miR-195 upregulated cell proliferation compared with the anti-ctrl group (Fig. 2A and B). In addition, miR-195-transfected cells exhibited lower colony formation ability compared with miR-ctrl-transfected cells. By contrast, anti-miR-195-transfected cells exhibited higher colony formation ability compared with anti-ctrl-transfected cells (Fig. 2C and D). These results indicate that miR-195 inhibited HepG2 cell proliferation and further suggests a tumor suppressive effect of miR-195 in HCC.

miR-195 induces cell cycle arrest and apoptosis in HCC. In order to further demonstrate the significance of the function of miR-195 in HCC cells, cells transfected with miR-195 were compared with cells transfected with miR-ctrl, and cells transfected with anti-miR-195 were compared with anti-ctrl to detect whether miR-195 exerted apoptotic effects in HCC. miR-195 overexpression resulted in a significant increase in the number of cells in the G1 phase of the cell cycle compared with miR-ctrl overexpression $(\mathrm{P}<0.05$; Fig. 3A) and the effect of miR-195 inhibitor transfection was to significantly reduce the number of HepG2 cells in the G1 phase of the cell cycle compared with control inhibitor transfection $(\mathrm{P}<0.05$; Fig. $3 \mathrm{~B})$. Furthermore, the results of cell apoptosis analysis demonstrate that miR-195 transfection significantly increased the number of cells in the early apoptotic stages compared with control transfection $(\mathrm{P}<0.05$; Fig. 3C); conversely, anti-miR-195 transfection significantly reduced the number of cells in early apoptosis compared with anti-control transfection ( $\mathrm{P}<0.05$; Fig. 3D). 
A

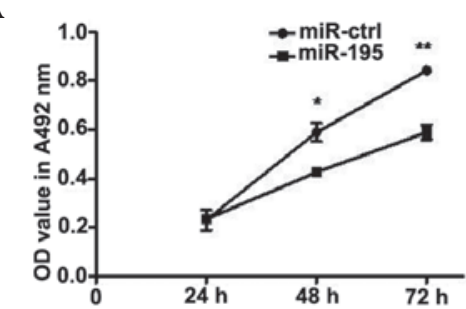

C

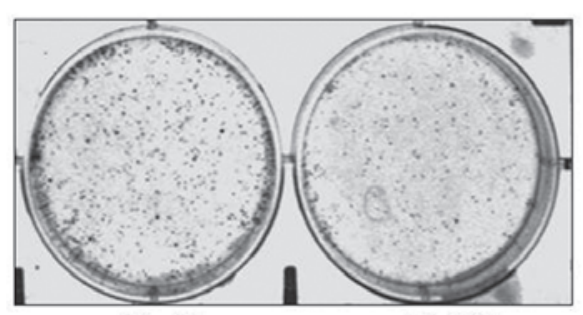

miR-ctr

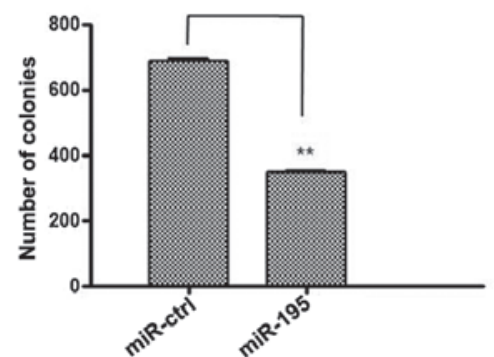

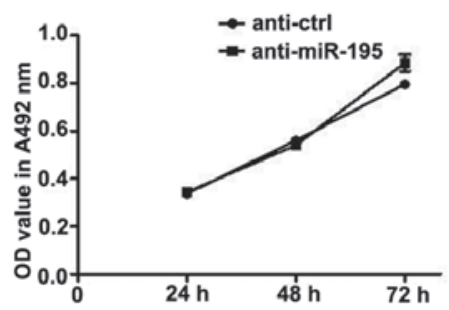

D

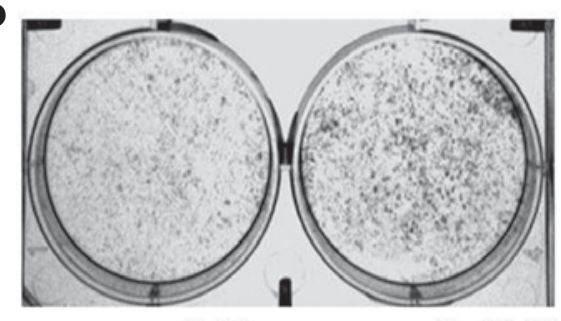

anti-ctrl

anti-miR-195

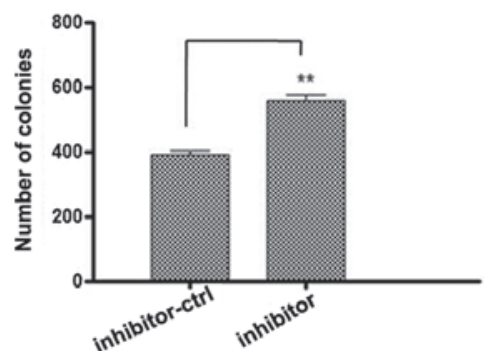

Figure 2. miR-195 inhibited hepatocellular carcinoma cell proliferation. (A and B) HepG2 cells were transfected with miR-195, miR-195-control, anti-miR-195 or anti-miR-195-control as indicated. Cell viability was measured by MTT assay. miR-195 transfection was shown to reduce cell growth compared with transfection with the control. However, the difference in cell growth between the two inhibitor groups (anti-miR-195 or anti-miR-195-control) was not marked. (C and D) HepG2 cells were transfected as above and cell proliferation was measured by colony formation assay. Colony formation ability was significantly reduced following miR-195 transfection, compared with control transfection. Furthermore, anti-miR-195 promoted significantly increased colony formation in HepG2 cells, compared with anti-miR-195-control. Data are presented as the mean \pm standard deviation of three independent experiments. ${ }^{*} \mathrm{P}<0.05$; ${ }^{* *} \mathrm{P}<0.01$. miR, microRNA; ctrl, control; OD, optical density.

A

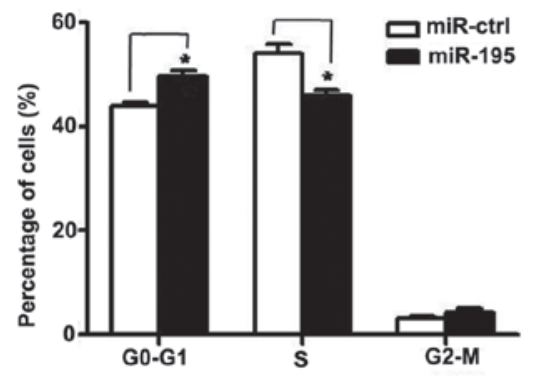

C

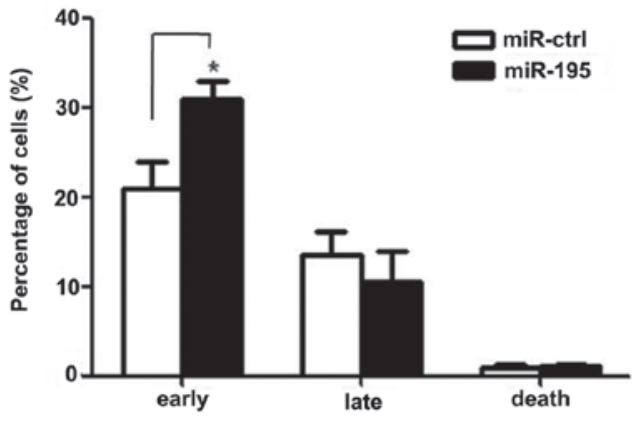

B

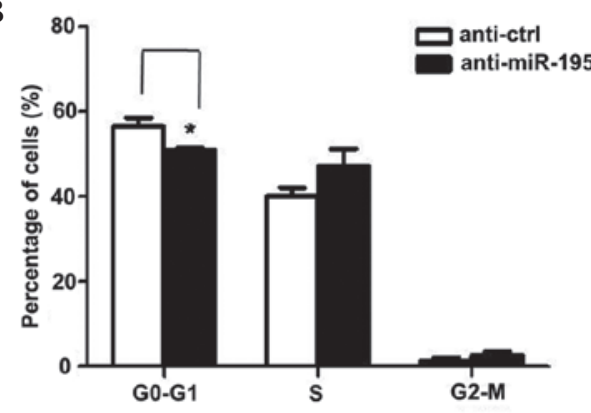

D

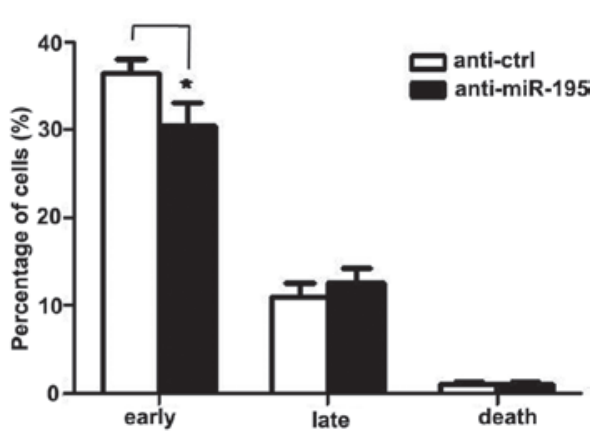

Figure 3. miR-195 affected the cell cycle and cell apoptosis. HepG2 cells were transfected with miR-195, miR-ctrl, anti-miR-195 or anti-ctrl. The percentage of cells at each stage of the cell cycle was measured after $48 \mathrm{~h}$. (A and B) miR-195 induced $\mathrm{G}_{0}-\mathrm{G}_{1} / \mathrm{S}$ phase cell cycle arrest in HepG2 cells. (C and D) HepG2 cells were transfected as above. After two days, the cells were harvested for cell apoptosis analysis, which indicated that miR-195 transfection significantly promoted HepG2 cell apoptosis compared with the control transfection. Results were obtained from three independent experiments. ${ }^{*} \mathrm{P}<0.05 ;{ }^{* *} \mathrm{P}<0.01$. miR, microRNA. 
A

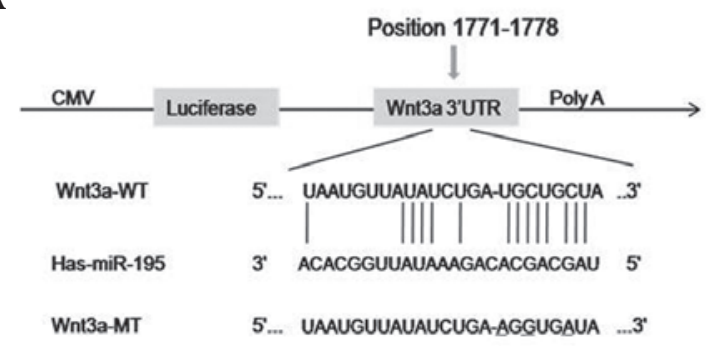

C

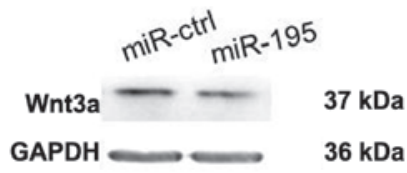

B
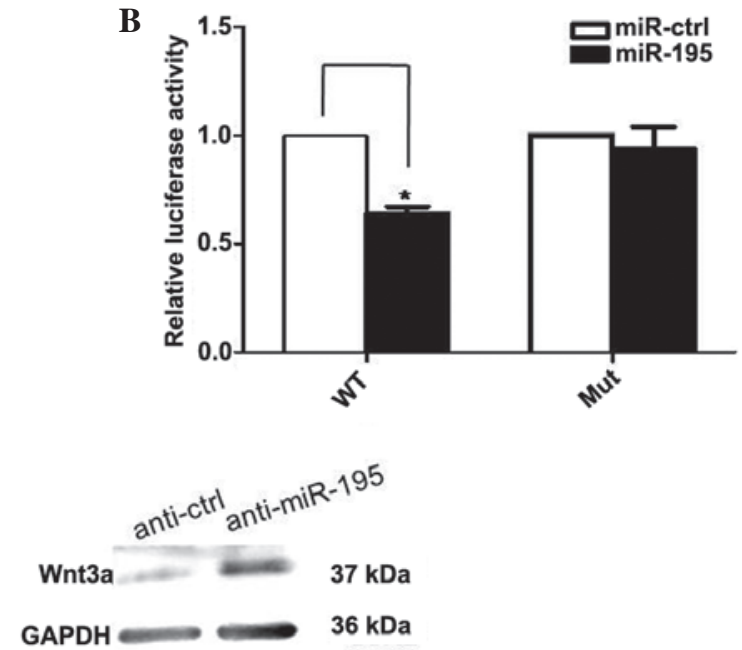

Figure 4. Wnt3a was a direct target of miR-195. (A) The miR-195-binding sequence in the 3'UTR of Wnt3a mRNA. A mutation was generated in the Wnt3a 3'UTR sequence in the complementary site for the seed region of miR-195, as shown. (B) HEK293 cells seeded in 96-well dishes were co-transfected with either the wild-type or mutant Wnt3a-3'UTR, together with miR-195 or pcDNA6.2-control. After 48 h, the relative luciferase values were measured and normalized by Renilla luciferase activity. The expression of Wnt3a was directly downregulated by miR-195. Data indicate the mean \pm standard deviation $(\mathrm{n}=3)$. Similar results were obtained from three independent experiments. ${ }^{*} \mathrm{P}<0.05$. (C) HepG2 cells were transfected with miR-195, miR-ctrl, anti-miR-195 or anti-ctrl. After $48 \mathrm{~h}$, the expression levels of Wnt3a were detected by western blot analysis. GAPDH served as an internal control. The result revealed that miR-195 transfection repressed the translation of Wnt3a protein in HepG2 cells, compared with miR-ctrl transfection, and that transfection with anti-miR-195 promoted the expression of Wnt3a, compared with anti-ctrl transfection. miR, microRNA; UTR, untranslated region; CMV, cytomegalovirus; WT, wild-type; ctrl, control.

Thus, miR-195 transfection induced G1 phase cell cycle arrest and promoted HCC cell apoptosis. These results indicate that miR-195 may exert a tumor suppressor function in HCC.

Wnt3a is a direct target of miR-195. To identify the molecular targets of miR-195, as miRNAs function mainly through posttranscriptional inhibition of target mRNAs via binding to the 3'UTR, an online miRNA target database TargetScan (http:// www.targetscan.org) was used to predict the target genes. Wnt3a has been indicated to be a potential target of miR-195 with a complementary 3'UTR binding site for the seed sequence of miR-195 (Fig. 4A). Co-transfection of miR-195 along with Wnt3a wild-type 3'UTR resulted in a significant reduction in luciferase activity compared with that of the control $(\mathrm{P}<0.05)$. However, co-transfection with miR-195 along with the Wnt3a-mut did not reduce luciferase activity (Fig. 4B). These results suggest that miR-195 directly targets Wnt3a. Additionally, western blot analysis was performed to measure the expression levels of Wnt3a protein in miR-195-transfected HCC cells and miR-195 transfection was found to reduce Wnt3a protein expression levels compared with miR-ctrl transfection. The expression levels of Wnt3a protein following transfection with anti-miR-195 or anti-ctrl were also determined (Fig. 4C). The expression levels of Wnt3a protein were significantly increased in the cells transfected with anti-miR-195 compared with the cells treated with anti-miRNA-control after $48 \mathrm{~h}$. The results revealed that the endogenously expressed Wnt3a was regulated by miR-195. These data demonstrate that Wnt3a is a direct target of miR-195.

In conclusion, these results suggest that overexpression of miR-195 inhibited HCC proliferation by repressing Wnt3a expression and that downregulation of miR-195 is important in the progression of HCC.

\section{Discussion}

Recent studies have shown that miRNAs exert wide effects through regulating the expression of a variety of genes in the majority of biological processes. Therefore, it is no surprise that $\mathrm{miRNAs}$ regulate the development of cancer. Investigating the role of individual miRNAs in cancer has generated great interest (20); the effects of differently expressed miRNAs may contribute to human carcinogenesis by regulating multivarious types of target gene expression (21). Recently, studies have demonstrated that miR-195 suppresses human glioma cell proliferation by directly targeting the 3'UTRs of cyclin D1 and cyclin E1 (22). miR-195 also exhibited an anti-apoptotic function in human colorectal cancer cells by targeting Bcl-2 (23).

In recent years, a number of signaling pathways have been found to be associated with HCC (24), including the AKT/mammalian target of rapamycin (25) mitogen-activated protein kinase kinase/extracellular signal-regulated kinase (26), c-Jun N-terminal kinase (27), nuclear factor-kappa B (28) and Wnt/ $\beta$-catenin signaling pathways (29). The Wnt/ $\beta$-catenin signaling pathway is vital in tumorigenesis and the progression of numerous types of tumor $(30,31)$. The Wnt signaling pathway was first associated with cancer when Wntla was identified as an oncogene in mouse breast cancer in 1982 (32). Wnt/ $\beta$-catenin signaling pathway-induced G1 phase progression through cyclin D1 and c-Myc transcriptional inductions has been considered to be a mediator between the cell cycle and Wnt signaling (33-36). Subsequent studies have revealed that cell proliferation, differentiation, apoptosis and other biological processes are affected by the Wnt signaling pathway (31). Multiple factors in the Wnt signaling pathway, including Wnt, $\beta$-catenin, adenomatosis polyposis coli, Axin 
and secreted frizzled-related protein 1, have been shown to be overexpressed in hepatocellular carcinoma cells $(37,38)$. The $\beta$-catenin/T-cell factor heterodimer activates $>60$ target genes of the Wnt signaling pathway, such as c-myc, c-jun and cyclin D1. These unusual activated oncogenes induce rapid proliferation in liver cells and may result in $\operatorname{HCC}(36,39,40)$.

In the present study, miR-195 overexpression was identified to directly target Wnt3a-induced G1 phase cell cycle arrest and promote apoptosis. Furthermore, the reduction in cell proliferation was marked. The data suggest that miR-195 is a potential diagnostic marker and that Wnt3a may be a key target in gene treatment of $\mathrm{HCC}$.

\section{Acknowledgements}

This study was supported by the National Natural Science Foundation of China (grant no. 81171398), the Special Major Scientific and Technological Research Projects of Shaanxi Province (grant no. 2010ZDKG-50) and the Program for Changjiang Scholars and Innovative Research Team in University (grant no. PCSIRT:1171).

\section{References}

1. Roberts LR: Sorafenib in liver cancer - just the beginning. N Engl J Med 359: 420-422, 2008.

2. Yamashita T and Wang XW: Cancer stem cells in the develop ment of liver cancer. J Clin Invest 123: 1911-1918, 2013.

3. Nordenstedt H, White DL and El-Serag HB: The changing pattern of epidemiology in hepatocellular carcinoma. Dig Liver Dis 42 (Suppl 3): S206-S214, 2010.

4. McGlynn KA and London WT: The global epidemiology of hepatocellular carcinoma: present and future. Clin Liver Dis 15: 223-243, 2011.

5. Long XH, Mao JH, Peng AF, et al: Tumor suppressive microRNA-424 inhibits osteosarcoma cell migration and invasion via targeting fatty acid synthase. Exp Ther Med 5: 1048-1052, 2013

6. Hiyoshi Y, Kamohara H, Karashima R, et al: MicroRNA-21 regulates the proliferation and invasion in esophageal squamous cell carcinoma. Clin Cancer Res 15: 1915-1922, 2009.

7. Moriyama T, Ohuchida K, Mizumoto K, et al: MicroRNA-21 modulates biological functions of pancreatic cancer cells including their proliferation, invasion, and chemoresistance. Mol Cancer Ther 8: 1067-1074, 2009.

8. Chen D, Zhang Y, Wang J, et al: MicroRNA-200c overexpression inhibits tumorigenicity and metastasis of $\mathrm{CD} 117^{+} \mathrm{CD} 44^{+}$ovarian cancer stem cells by regulating epithelial-mesenchymal transition. J Ovarian Res 6: 50, 2013.

9. Coulouarn C, Factor VM, Andersen JB, Durkin ME and Thorgeirsson SS: Loss of miR-122 expression in liver cancer correlates with suppression of the hepatic phenotype and gain of metastatic properties. Oncogene 28: 3526-3536, 2009.

10. Liang L, Wong CM, Ying Q, et al: MicroRNA-125b suppressed human liver cancer cell proliferation and metastasis by directly targeting oncogene LIN28B2. Hepatology 52: 1731-1740, 2010.

11. Liu AM, Poon RT and Luk JM: MicroRNA-375 targets Hippo-signaling effector YAP in liver cancer and inhibits tumor properties. Biochem Biophys Res Comm 394: 623-627, 2010.

12. Meng F, Henson R, Wehbe-Janek H, et al: MicroRNA-21 regulates expression of the PTEN tumor suppressor gene in human hepatocellular cancer. Gastroenterology 133: 647-658, 2007.

13. Su H, Yang JR, Xu T, et al: MicroRNA-101, down-regulated in hepatocellular carcinoma, promotes apoptosis and suppresses tumorigenicity. Cancer Res 69: 1135-1142, 2009.

14. Zhang J, Luo N, Luo Y, et al: microRNA-150 inhibits human CD133-positive liver cancer stem cells through negative regulation of the transcription factor c-Myb. Int J Oncol 40: 747-756, 2012.
15. Ichimi T, Enokida H, Okuno Y, et al: Identification of novel microRNA targets based on microRNA signatures in bladder cancer. International Journal of Cancer 125: 345-352, 2009.

16. Soon PSH, Tacon LJ, Gill AJ, et al: miR-195 and miR-483-5p identified as predictors of poor prognosis in adrenocortical cancer. Clin Cancer Res 15: 7684-7692, 2009.

17. Li D, Zhao Y, Liu C, et al: Analysis of MiR-195 and MiR-497 expression, regulation and role in breast cancer. Clin Cancer Res 17: 1722-1730, 2011

18. Guo J, Miao Y, Xiao B, et al: Differential expression of microRNA species in human gastric cancer versus non-tumorous tissues. J Gastroenterol Hepatol 24: 652-657, 2009.

19. Cadigan KM and Nusse R: Wnt signaling: a common theme in animal development. Genes Dev 11: 3286-3305, 1997.

20. Majid S, Dar AA, Saini S, et al: MicroRNA-23b functions as a tumor suppressor by regulating Zeb1 in bladder cancer. PloS One 8: e67686, 2013.

21. Volinia S, Calin GA, Liu CG, et al: A microRNA expression signature of human solid tumors defines cancer gene targets. Proc Natl Acad Sci USA 103: 2257-2261, 2006.

22. Hui W, Yuntao L, Lun L, et al: MicroRNA-195 inhibits the proliferation of human glioma cells by directly targeting cyclin D1 and cyclin E1. PloS One 8: e54932, 2013.

23. Liu L, Chen L, Xu Y, Li R and Du X: microRNA-195 promotes apoptosis and suppresses tumorigenicity of human colorectal cancer cells. Biochem Biophys Res Commun 400: 236-240, 2010.

24. Fatima S, Lee NP and Luk JM: Dickkopfs and Wnt/3-catenin signalling in liver cancer. World J Clin Oncol 2: 311-325, 2011.

25. Calvisi DF, Wang C, Ho C, et al: Increased lipogenesis, induced by AKT-mTORC1-RPS6 signaling, promotes development of human hepatocellular carcinoma. Gastroenterology 140: 1071-1083, 2011.

26. Caja L, Sancho P, Bertran E, et al: Overactivation of the $\mathrm{MEK} / \mathrm{ERK}$ pathway in liver tumor cells confers resistance to TGF- $\beta$-induced cell death through impairing up-regulation of the NADPH oxidase NOX4. Cancer Res 69: 7595-7602, 2009.

27. Chang Q, Zhang Y, Beezhold KJ, et al: Sustained JNK1 activation is associated with altered histone $\mathrm{H} 3$ methylations in human liver cancer. J Hepatol 50: 323-333, 2009.

28. He Y, Zhang H, Yin J, et al: I $\mathrm{B} \alpha$ gene promoter polymorphisms are associated with hepatocarcinogenesis in patients infected with hepatitis B virus genotype C. Carcinogenesis 30: 1916-1922, 2009.

29. Whittaker S, Marais R and Zhu A: The role of signaling pathways in the development and treatment of hepatocellular carcinoma. Oncogene 29: 4989-5005, 2010.

30. Lu D, Zhao Y, Tawatao R, et al: Activation of the Wnt signaling pathway in chronic lymphocytic leukemia. Proc Natl Acad Sci USA 101: 3118-3123, 2004.

31. Ilyas M: Wnt signalling and the mechanistic basis of tumour development. J Pathol 205: 130-144, 2005.

32. Nusse R and Varmus HE: Many tumors induced by the mouse mammary tumor virus contain a provirus integrated in the same region of the host genome. Cell 31: 99-109, 1982.

33. Gougelet A and Colnot S: A Complex Interplay between Wnt $/ \beta$-Catenin Signalling and the Cell Cycle in the Adult Liver. Int J Hepatol 2012: 816125, 2012.

34. He TC, Sparks AB, Rago C, et al: Identification of c-MYC as a target of the APC pathway. Science 281: 1509-1512, 1998.

35. Shtutman M, Zhurinsky J, Simcha I, et al: The cyclin D1 gene is a target of the $\beta$-catenin/LEF-1 pathway. Proc Natl Acad Sci USA 96: 5522-5527, 1999 .

36. Tetsu $\mathrm{O}$ and McCormick F: $\beta$-Catenin regulates expression of cyclin D1 in colon carcinoma cells. Nature 398: 422-426, 1999.

37. Kaur P, Mani S, Cros MP, et al: Epigenetic silencing of sFRP1 activates the canonical Wnt pathway and contributes to increased cell growth and proliferation in hepatocellular carcinoma. Tumor Biol 33: 325-336, 2012.

38. Guan CN, Chen XM, Lou HQ, et al: Clinical Significance of Axin and $\beta$-catenin Protein Expression in Primary Hepatocellular Carcinomas. Asian Pac J Cancer Prev 13: 677-681, 2012.

39. Loeppen S, Koehle C, Buchmann A and Schwarz M: A $\beta$-catenin-dependent pathway regulates expression of cytochrome P450 isoforms in mouse liver tumors. Carcinogenesis 26: 239-248, 2005 .

40. Tien LT, Ito M, Nakao M, et al: Expression of $\beta$-catenin in hepatocellular carcinoma. World J Gastroenterol 11: 2398-2401, 2005. 